\title{
Tailor-made inflammation: how neutrophil serine proteases modulate the inflammatory response
}

\author{
Kai Kessenbrock • Therese Dau • Dieter E. Jenne
}

Received: 15 September 2009 /Revised: 6 July 2010 /Accepted: 2 August 2010 /Published online: 31 August 2010

(C) The Author(s) 2010. This article is published with open access at Springerlink.com

\begin{abstract}
Neutrophil granulocytes are important mediators of innate immunity, but also participate in the pathogenesis of (auto)inflammatory diseases. Neutrophils express a specific set of proteolytic enzymes, the neutrophil serine proteases (NSPs), which are stored in cytoplasmic granules and can be secreted into the extra- and pericellular space upon cellular activation. These NSPs, namely cathepsin G (CG), neutrophil elastase (NE), and proteinase 3 (PR3), have early been implicated in bacterial defense. However, NSPs also regulate the inflammatory response by specifically altering the function of cytokines and chemokines. For instance, PR3 and NE both inactivate the anti-inflammatory mediator progranulin, which may play a role in chronic inflammation. Here, we provide a concise update on NSPs as modulators of inflammation and discuss the biological and pathological significance of this novel function of NSPs. Mounting evidence support an important proinflammatory function for PR3, which may have been underestimated in the past.
\end{abstract}

Keywords Neutrophil serine proteases - Inflammation . Neutrophil elastase · Proteinase $3 \cdot$ Cathepsin G . Progranulin

Electronic supplementary material The online version of this article (doi:10.1007/s00109-010-0677-3) contains supplementary material, which is available to authorized users.

\footnotetext{
K. Kessenbrock $(\bowtie)$

Department of Anatomy, University of California,

513 Parnassus Avenue,

San Francisco, CA 94143-0452, USA

e-mail: kai.kessenbrock@ucsf.edu

T. Dau $\cdot$ D. E. Jenne

Max-Planck-Institute of Neurobiology,

Department of Neuroimmunology,

Am Klopferspitz 18,

82152 Martinsried, Germany
}

\section{Introduction}

Neutrophil granulocytes make up the majority of all leukocytes in peripheral, human blood, where they circulate through the body and swiftly react on danger signals from injured or infected tissues. Once infection or wounding occurs, neutrophils are one of the first cell types that infiltrate the harmed tissue, where they release antimicrobial factors and reactive oxygen species (ROS) to kill pathogens and disinfect the injured tissue. Neutrophils contribute to inflammation and bacterial defense mainly via three distinct cell biological mechanisms, namely by phagocytosis, degranulation or the formation of neutrophil extracellular traps (NETs) [1]. Bacterial infections are usually battled via phagocytosis of the pathogens, i.e., the receptor-mediated uptake of bacteria followed by degradation of the pathogen inside the phagolysosome. Neutrophils are pre-charged with antimicrobial factors such as neutrophil serine proteases (NSPs), which are stored in cytoplasmic organelles, the so-called azurophilic granules, ready to be released into the extracellular space by degranulation. When neutrophils encounter bacteria, they can also undergo an alternative cell death program leading to the extrusion of their own nuclear chromatin to form NETs, extracellular chromatin fibers decorated with antimicrobial proteases that trap and kill bacteria even after neutrophil cell death [2]. However, uncontrolled neutrophil activation can lead to chronic inflammatory conditions such as autoimmune diseases or hypersensitivity reactions. For instance, neutrophil activation by autoantibodies in autoimmune smallvessel vasculitis promotes the formation of NETs, which are deposited in the kidneys of these patients and are likely to drive the disease process and the autoimmune response against myeloperoxidase and PR3 present on these NETs [3]. Recent studies have clarified that the physiological role 
of NSPs is not limited to the degradation of microbes, but also involves specific immunoregulatory functions that affect the cascade of events during the inflammatory reaction [4]. In this minireview, we provide a concise update on the role of NSPs as inflammatory mediators and discuss the biological and pathological significance of these events.

\section{Neutrophil serine proteases}

Neutrophils express several classes of proteases including matrix metalloproteinases such as matrix metalloproteinase (MMP)-8 and -9 , cysteine proteases such as caspases or calpain, and NSPs. Serine proteases are a class of proteases with the amino acid serine as the key residue of their enzymatic center, which initiates the proteolytic cleavage of specific target proteins, the protease substrates. The serine proteases of neutrophils cathepsin $\mathrm{G}(\mathrm{CG})$, neutrophil elastase (NE), and proteinase 3 (PR3) are crucially involved in bacterial defense [5], but also mediate regulatory functions that shape the inflammatory response [4]. While PR3 and $\mathrm{NE}$ are very similar in their substrate specificity supporting a potentially redundant function for these enzymes, CG usually targets a different set of substrate proteins.

Unregulated extracellular proteolysis would be detrimental to the entire organism. Hence, the liver constantly produces copious amounts of physiological serine protease inhibitors, such as $\alpha 1$-antitrypsin ( $\alpha 1-\mathrm{AT}), \alpha 1$-antichymotrypsin, and $\alpha 2$-macroglobulin, which are distributed throughout the body in the plasma and interstitial fluids and efficiently remove NSPs and other proteases after their release by complexation and delivery to lysosomes of phagocytic cells. Moreover, there are tissue specific inhibitors of NSPs such as elafin or secretory leukocyte protease inhibitor (SLPI), which control serine protease activity in interstitial fluids. Disruption of the balance between protease and their inhibitors as it occurs in patients with genetic $\alpha 1$-AT deficiency leads to the development of lung emphysema and certain autoimmune disorders [6]. This reflects the involvement of NSPs in the progression of inflammatory conditions and tissue breakdown.

\section{NSPs control inflammatory reactions}

The role of NSPs in inflammation has been controversial for several years. Most knowledge about the function of NSPs has been gained from in vitro studies using purified enzymes. Initial findings that NSPs cleave extracellular matrix components suggested that these enzymes might facilitate the egress of leukocytes from blood vessels and pave the way towards the sites of inflammation. More recent reports have provided interesting in vitro results showing that NSPs cleave several chemokines and cytokines, which leads to enhancement or abolishment of the function of these immune mediators [7, 8]. Some of these studies analyzed ex vivo specimens by comparing chemokine or cytokine cleavage in specimens from patients suffering from inflammatory conditions with those from healthy control individuals (summarized in Supplementary Table 1). However, the biological significance of these data remains to be addressed using adequate experimental in vivo models in order to elucidate if a substrate is in fact targeted by the protease under physiological conditions.

The recent generation of NSP-deficient mice has formed the basis for the in vivo evaluation of the role of these proteases during inflammation. For instance, NE-deficient animals have been used to determine whether this enzyme is critical for neutrophil extravasation during inflammation as suggested by previous in vitro results. However, these papers have yielded conflicting results regarding the effect of NE-deficiency on neutrophil extravasation to sites of inflammation, since NE may or may not be important for leukocyte migration across the basement membrane $[9,10]$. One of the first in vivo findings that supported an immunoregulatory role of NSPs were made in mice deficient in dipeptidyl peptidase I (DPPI), another protease that mediates the conversion of NSPs from their inactive precursors (zymogen) into the enzymatically active protease by removal of an N-terminal pro-peptide [11]. Neutrophils of DPPI-deficient mice showed very little NSP enzymatic activity and demonstrated strongly decreased infiltration to the sites of inflammation in an experimental arthritis model, although neutrophil migration in vivo appeared generally normal in these mice. Of importance, this was accompanied by decreased local production of inflammatory cytokines. These findings suggest that NSPs modulate cellular events such as neutrophil activation and chemokine production, while at least in this setting they are not directly necessary for the crossing of the endothelial basement membrane during neutrophil diapedesis.

Cellular activation of neutrophil granulocytes during the inflammatory response leads to the substantial release of NSPs into the extracellular space, where the proteolytic activity of these enzymes is tightly regulated by local protease inhibitors (Fig. 1a). There are several ways how NSPs can be active despite the presence of protease inhibitors. For instance, previous studies have revealed that NSPs compartmentalize to the cell surface of neutrophils where they may be shielded from inhibitor binding and therefore fully active [12]. NSPs are also present on extracellular chromatin fibers called NETs [2, 3]. It remains to be determined whether NSPs on NETs are enzymatically active and whether their interaction with endogenous inhibitors is altered. Moreover, inhibitors of NSPs can be degraded by other proteases released from activated neutrophils such as MMP-9, which degrades the serpin 
Fig. 1 NSPs regulate inflammatory processes via multiple pathways. a Neutrophil activation by proinflammatory stimuli lead to increased externalization of NSPs. Activated neutrophils (red) release NSPs by degranulation, or formation of NETs. Certain fractions of NSPs are compartmentalized to cell surface receptors and stay in the pericellular environment. The function of NSPs is controlled by the balance between proteases and protease inhibitors circulating in blood and interstitial fluids. b Extracellular PR3 converts IL-8 released from neutrophils and endothelial cells by $\mathrm{N}$-terminal truncation (cleavage sequence is indicated) into a more bioactive chemokine. This may potently enhance the recruitment of further neutrophils to the site of inflammation. c PR3 acts as a converting enzyme of pro$\mathrm{TNF} \alpha$, the membrane-attached precursor of the potent proinflammatory cytokine TNF $\alpha$.

This may be a crucial step in the establishment of an inflammatory milieu. d Neutrophils extravasating from the vasculature are initially controlled by anti-inflammatory PGRN. PR3 and NE cooperatively enhance neutrophil activation by specifically degrading inflammationsuppressing PGRN. e CG interacts with surface integrins during the adhesion of neutrophils to immobilized immune complexes, where it promotes integrin clustering, cytoskeletal rearrangements, and subsequently the release of neutrophil attracting chemokines
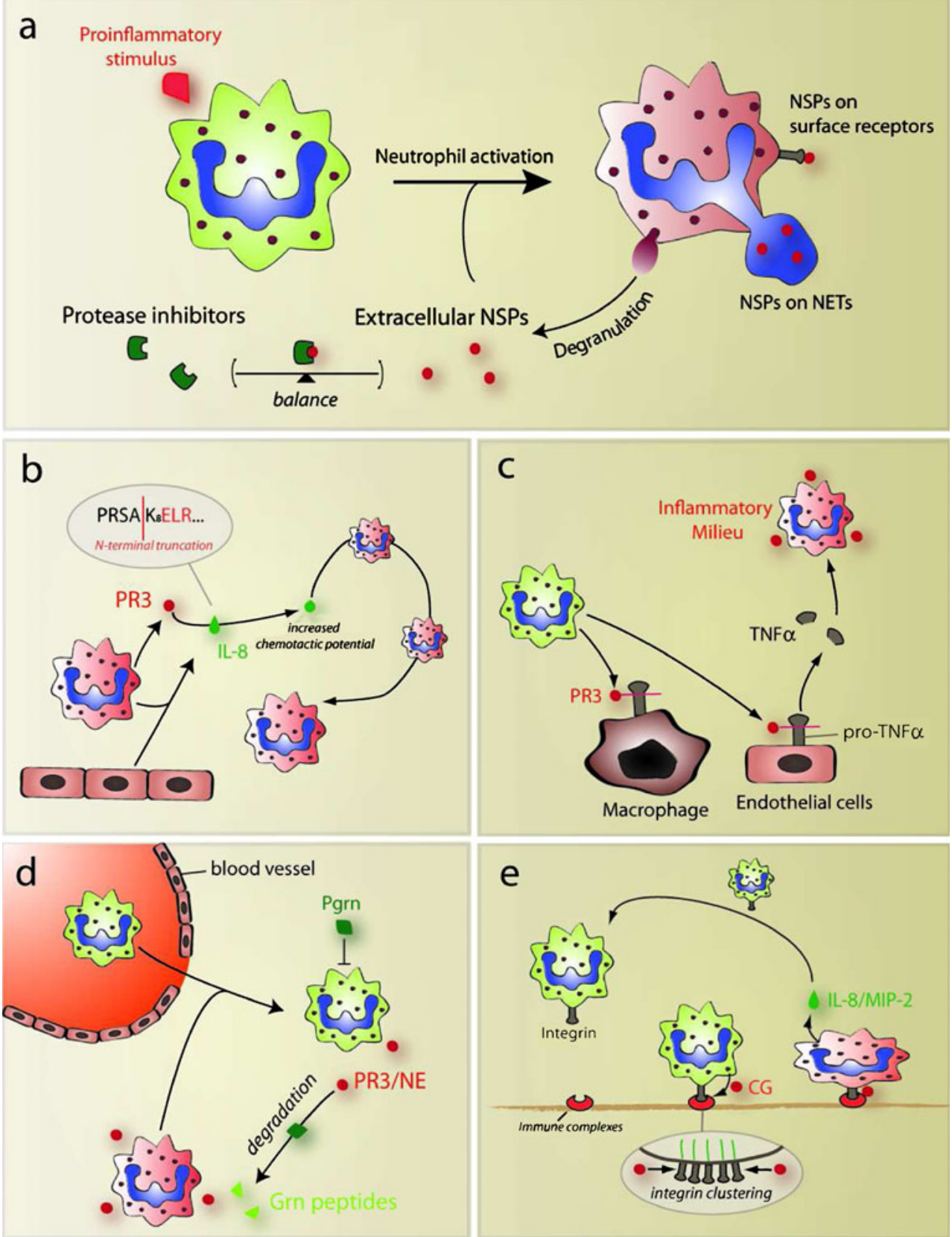

$\alpha 1-P I$ and thus prolongs local NE proteolytic activity [13]. Hence, the pericellular microenvironment around a neutrophil may be depleted from local inhibitors leading to increased NSP activity at the site of inflammation.

But how do NSPs regulate the course of an inflammatory response on the molecular level? In the following parts, we will highlight selected actions that involve NSP function during the inflammatory response.

\section{Regulation of chemokine function}

The coordination of leukocyte migration to a damaged tissue is essentially mediated by a concentration gradient of chemokines, which is sensed by the migrating cell using $\mathrm{G}$ protein-coupled receptors. Extracellular proteases modulate chemokine function in several ways, which may lead to abrogation or enhancement of chemokine function, and sometimes alters the bioavailability of the chemokine.

Proteolytic abrogation of chemokine function often leads to an inflammation-dampening effect or changes the subsequent recruitment of immune cells. The stromaderived factor $1 \alpha(\mathrm{SDF}-1 \alpha)$ is an important lymphocyteattracting chemokine. The proteolytic cleavage of SDF- $1 \alpha$ by $\mathrm{NE}$ on the endothelium during neutrophil extravasation inactivates the chemokine and leads to decreased secondary recruitment of lymphocytes to the site of inflammation. 
Therefore, inactivation of SDF- $1 \alpha$ by NE seems to be an important step that controls the temporal pattern of immune cell trafficking to inflammatory sites [14].

A common mechanism of chemokine alteration that frequently involves NSP function is the proteolytic truncation at the $\mathrm{N}$-terminus of the chemokine. In many cases, this removes several amino acids $\mathrm{N}$-terminal to the chemotactic motif without destroying its biological function, but rather leading to enhanced chemotactic potential of the molecule [8]. Interleukin 8 (IL-8) is one prominent neutrophil-attracting chemokine that is cleaved by all three members of NSPs with differential effects on its biological function. Interestingly, while $\mathrm{CG}$ and $\mathrm{NE}$ mediated Nterminal truncation of IL-8 leads to inactivation of the chemokine, PR3 proteolytically converts IL-8 into a more potent neutrophil activating version [15]. This may have important implications during the recruitment of inflammatory cells to the site of inflammation (Fig. 1b). However, the biological significance of this proteolytic conversion of chemokines remains to be evaluated in vivo. While there is no direct homolog of IL-8 in mice, the respective IL8 receptor homolog is known to be activated strongest by macrophage inflammatory protein 2 (MIP-2), and by the approximately tenfold less potent chemokine $\mathrm{KC}$ [16]. Hence, analyzing the cleavage of MIP- 2 and $\mathrm{KC}$ in mice, for instance in NSP-depleted mice, will provide a useful tool to address the biological significance of proteolytic modulation of IL-8.

\section{Conversion of inflammatory mediators}

The posttranslational conversion of cytokine precursors by extracellular proteases determines the activation of cytokines and the time frame of their activity. For instance, all three members of the NSPs have been shown to inactivate the soluble IL-6 receptor, thus interfering with IL-6 signaling, which may contribute to the inflammatory condition in patients suffering from cystic fibrosis [17]. Another key proinflammatory mediator is tumor necrosis factor $\alpha(\mathrm{TNF} \alpha)$ that is produced as a membrane-attached precursor and activated by proteolytic conversion after which the active form of TNF $\alpha$ is solubilized. This is mostly carried out by a metalloproteinase, named TNF $\alpha$ converting enzyme (TACE, ADAM17) [18]. Interestingly, also PR3 seems to contribute to TNF $\alpha$ conversion for example in pneumonia, as indicated by experiments using alveolar macrophages, which, although they show no expression of PR3, seem to carry significant amounts of PR3 on their cell surface [19]. This might be an important function of PR3 during the establishment of an inflammatory milieu (Fig. 1c). Moreover, PR3 proteolytically activates further inflammatory cytokines including IL-1 $\beta$ [20], IL-18 [21], as well as IL-32, a recently discovered cytokine that may play a crucial role during lung inflammation by triggering the release of neutrophil-attracting IL8 from epithelial cells [22]. Although caspase-1 is probably the most important IL-1 $\beta$-activating protease, results from DPPI-deficient mice, which lack NSP enzymatic activity, argue for an important role of PR3 in the conversion of this potent inflammatory mediator in a mouse model for rheumatoid arthritis [23]. The fact that all these cytokines are selectively converted by PR3, but not by CG or NE, points towards a distinct proinflammatory role of PR3 during the establishment of an inflammatory environment. However, these findings are mainly based on in vitro studies and remain to be corroborated by in vivo experiments to determine the biological relevance of PR3-mediated conversion of these cytokines.

Progranulin (PGRN; also known as granulin-epithelin precursor) is an important regulator of the wound-healing response [24]. Zhu and co-workers have described that PGRN potently suppresses the adhesion-dependent oxidative burst and release of proteases by neutrophils in the presence $\mathrm{TNF} \alpha$, however, it loses its anti-inflammatory activity after cleavage by NE [25]. Recent studies using PR3/NE double-deficient mice revealed that PGRN is degraded by both PR3 and NE during neutrophil activation in vitro and immune complex-mediated inflammation in vivo [26]. In the absence of PR3 and NE, anti-inflammatory PGRN was not degraded any more leading to significantly higher PGRN levels during the inflammatory response. This may inhibit the activation of infiltrating neutrophils and most likely leads to significantly decreased feedback recruitment of inflammatory cells, which explains the impaired inflammatory response to immune complex deposition in PR3/NE-deficient mice (Fig. 1d). This mechanism may play an important role in the establishment and control of the chronic inflammation. Interestingly, lossof-function mutations in the $P G R N$ gene are associated with the development of neurodegenerative disorders such as dementia, Alzheimer, and Parkinson disease [27]. Indeed, recent studies using PGRN-deficient mice suggest that decreased levels of PGRN in these patients leads to a chronic, subtle inflammatory condition, which may finally manifest in age-related neurodegenerative disease [28]. The details about the exact molecular pathway of the antiinflammatory effects linked with PGRN yet remains to be elucidated.

NSPs engage surface receptors

As mentioned earlier, NSPs often bind to surface receptors, once they are externalized by activated neutrophils. This can create an auto- or paracrine effect, since several receptors transduce signals that promote neutrophil activation or alter epithelial cell function. For instance, CG 
appears to trigger integrin clustering on neutrophils interacting with immobilized immune complexes, which leads to cytoskeletal rearrangements, increased ROS production, and secretion of chemokines such as MIP-2 [29]. These findings suggest an important role of CG during immune complex-induced inflammation (Fig. 1e). However, the exact molecular mechanism as to how CG modulates integrin clustering on the cell surface remains elusive.

Another intriguing pathway of NSP-mediated inflammation is based on the engagement of protease-activated receptors (PARs), which are G protein-coupled receptors mostly expressed on platelets and epithelial cells that are activated by proteolytic cleavage of the extracellular domain. All three NSPs have been implicated in activating PAR-2 on human oral epithelial cells and gingival fibroblasts, which resulted in the release of proinflammatory mediators such as IL-8 [30], suggesting that PAR-activation by NSPs may contribute to the inflammatory process for example during peridontitis. Moreover, NE and PR3, but not CG, seem to be involved in activating PAR-1 and PAR-2 on epithelial cells leading to downregulation of epithelial barrier function and increased leukocyte transmigration, a process that may be important in the pathogenesis of inflammatory bowel disease [31]. Although NSPs may be important activators of PARs, many aspects of this relationship remain elusive. Here again, most findings are based on in vitro studies and would require further confirmation in the living animal. For instance, PAR-2 is known to be expressed on neutrophils, however, it is still unclear whether NSPs externalized by stimulated neutrophils promote an autocrine feedback on neutrophil activation via PAR-2.

The toll-like receptors (TLRs) represent another class of intra- and extracellular membrane-anchored molecules that sense inflammatory stimuli such as bacterial DNA or lipopolysaccharide (LPS) and mediate cellular activation via activation of the nuclear factor $\mathrm{KB}$ pathway. Interestingly, NE has been found to activate TLR- 4 , the surface receptor for LPS, leading to release of IL-8 by a human embryonic kidney cell line [32]. This process appears to depend on proteolytic activity as it was inhibited by phenylmethylsulphonyl fluoride, a broad spectrum serine protease inhibitor. However, the exact molecular pathway of TLR-4 activation by NE needs yet to be addressed and, although NSPs can modulate the function of integrins, PARs, or TLRs, there is yet no direct evidence that NSPs actually bind to these classes of cell surface molecules.

\section{Synergistic effects}

Many of the inflammatory pathways modulated by NSPs are interconnected, so that, hypothetically, NSPs may work synergistically to accelerate the defense reaction. For instance, NSPs mediate the release of IL- 8 by engaging several surface receptors such as TLRs, PARs, or integrins $[29,30,32]$. The bioactivity of IL-8 may then be enhanced by PR3-mediated truncation of the chemokine N-terminus [15], leading to increased recruitment of more neutrophils to the site of inflammation. Moreover, the generation of active TNF $\alpha$ by PR3 triggers the externalization of further NSPs by neutrophil granulocytes. Likewise, PGRN inhibits the release of NSPs by neutrophils [25], thus the degradation of PGRN by PR3 and NE may drive neutrophils to release additional NSPs. Furthermore, NSPs often trigger increased production of ROS by neutrophils, e.g., via the promotion of integrin clustering or by degrading anti-inflammatory PGRN $[26,29]$. Given that these oxidants inactivate physiological protease inhibitors [33], NSPs indirectly prolong the time frame of their actions by promoting ROS production.

\section{Conclusion and outlook}

Although NSPs sometimes inactivate certain inflammatory cytokines and chemokines, the function of NSPs generally promotes rather than suppresses the inflammatory response. Especially the relevance of PR3 as a proinflammatory mediator may have been underestimated in the past. Most of the target molecules have been identified by in vitro studies, hence the biological significance of these pathways often remains to be confirmed using in vivo using appropriate animal models. The three neutrophil enzymes $\mathrm{CG}, \mathrm{NE}$, and PR3 seem to synergize with each other during the establishment of an inflammatory milieu. Thus, the generation of NSP inhibitors specific for all three NSPs may be a potent inflammation-suppressing drug that could be used to treat patients with chronic inflammatory disorders.

Acknowledgments We acknowledge support by grants (SFB571, A4) from the Deutsche Forschungsgemeinschaft and by fellowships from the Susan G. Komen and Alexander von Humboldt Foundations.

Conflict of interest The authors declare that there is no conflict of interest.

Open Access This article is distributed under the terms of the Creative Commons Attribution Noncommercial License which permits any noncommercial use, distribution, and reproduction in any medium, provided the original author(s) and source are credited.

\section{References}

1. Nathan C (2006) Neutrophils and immunity: challenges and opportunities. Nat Rev Immunol 6:173-182

2. Brinkmann V, Reichard U, Goosmann C, Fauler B, Uhlemann Y, Weiss DS, Weinrauch Y, Zychlinsky A (2004) Neutrophil extracellular traps kill bacteria. Science 303:1532-1535 
3. Kessenbrock K, Krumbholz M, Schönermarck U, Back W, Gross WL, Werb Z, Gröne HJ, Brinkmann V, Jenne DE (2009) Netting neutrophils in autoimmune small-vessel vasculitis. Nat Med 15:623-625

4. Pham CT (2006) Neutrophil serine proteases: specific regulators of inflammation. Nat Rev Immunol 6:541-550

5. Segal AW (2005) How neutrophils kill microbes. Annu Rev Immunol 23:197-223

6. Stoller JK, Aboussouan LS (2005) Alpha1-antitrypsin deficiency. Lancet 365:2225-2236

7. Bank U, Ansorge S (2001) More than destructive: neutrophilderived serine proteases in cytokine bioactivity control. J Leukoc Biol 69:197-206

8. Mortier A, Van Damme J, Proost P (2008) Regulation of chemokine activity by posttranslational modification. Pharmacol Ther 120:197-217

9. Hirche TO, Atkinson JJ, Bahr S, Belaaouaj A (2004) Deficiency in neutrophil elastase does not impair neutrophil recruitment to inflamed sites. Am J Respir Cell Mol Biol 30:576-584

10. Young RE, Thompson RD, Larbi KY, La M, Roberts CE, Shapiro SD, Perretti M, Nourshargh S (2004) Neutrophil elastase (NE)-deficient mice demonstrate a nonredundant role for NE in neutrophil migration, generation of proinflammatory mediators, and phagocytosis in response to zymosan particles in vivo. J Immunol 172:4493-4502

11. Adkison AM, Raptis SZ, Kelley DG, Pham CT (2002) Dipeptidyl peptidase I activates neutrophil-derived serine proteases and regulates the development of acute experimental arthritis. J Clin Invest 109:363-371

12. Campbell EJ, Campbell MA, Owen CA (2000) Bioactive proteinase 3 on the cell surface of human neutrophils: quantification, catalytic activity, and susceptibility to inhibition. J Immunol 165:3366-3374

13. Liu Z, Zhou X, Shapiro SD, Shipley JM, Twining SS, Diaz LA, Senior RM, Werb Z (2000) The serpin alpha1-proteinase inhibitor is a critical substrate for gelatinase B/MMP-9 in vivo. Cell 102:647-655

14. Rao RM, Betz TV, Lamont DJ, Kim MB, Shaw SK, Froio RM, Baleux F, Arenzana-Seisdedos F, Alon R, Luscinskas FW (2004) Elastase release by transmigrating neutrophils deactivates endothelial-bound SDF-1alpha and attenuates subsequent $\mathrm{T}$ lymphocyte transendothelial migration. J Exp Med 200:713-724

15. Padrines M, Wolf M, Walz A, Baggiolini M (1994) Interleukin8 processing by neutrophil elastase, cathepsin $\mathrm{G}$ and proteinase-3. FEBS Lett 352:231-235

16. Lee J, Cacalano G, Camerato T, Toy K, Moore MW, Wood WI (1995) Chemokine binding and activities mediated by the mouse IL-8 receptor. J Immunol 155:2158-2164

17. McGreal EP, Davies PL, Powell W, Rose-John S, Spiller OB, Doull I, Jones SA, Kotecha S (2010) Inactivation of IL-6 and soluble IL-6 receptor by neutrophil derived serine proteases in cystic fibrosis. Biochim Biophys Acta 1802:649-658

18. Black RA, Rauch CT, Kozlosky CJ, Peschon JJ, Slack JL, Wolfson MF, Castner BJ, Stocking KL, Reddy P, Srinivasan S, Nelson N, Boiani N, Schooley KA, Gerhart M, Davis R, Fitzner JN, Johnson RS, Paxton RJ, March CJ, Cerretti DP (1997) A metalloproteinase disintegrin that releases tumour-necrosis factoralpha from cells. Nature 385:729-733

19. Armstrong L, Godinho SI, Uppington KM, Whittington HA, Millar AB (2009) Tumour necrosis factor-alpha processing in interstitial lung disease: a potential role for exogenous proteinase3. Clin Exp Immunol 156:336-343
20. Coeshott C, Ohnemus C, Pilyavskaya A, Ross S, Wieczorek M, Kroona H, Leimer AH, Cheronis J (1999) Converting enzymeindependent release of tumor necrosis factor alpha and IL-1beta from a stimulated human monocytic cell line in the presence of activated neutrophils or purified proteinase 3. Proc Natl Acad Sci USA 96:6261-6266

21. Sugawara S, Uehara A, Nochi $T$, Yamaguchi $T$, Ueda $H$, Sugiyama A, Hanzawa K, Kumagai K, Okamura H, Takada H (2001) Neutrophil proteinase 3-mediated induction of bioactive IL-18 secretion by human oral epithelial cells. J Immunol 167:6568-6575

22. Kim S, Lee S, Her E, Bae S, Choi J, Hong J, Jaekal J, Yoon D, Azam T, Dinarello CA, Kim S (2008) Proteinase 3-processed form of the recombinant IL-32 separate domain. BMB Rep 41:814-819

23. Joosten LA, Netea MG, Fantuzzi G, Koenders MI, Helsen MM, Sparrer H, Pham CT, van der Meer JW, Dinarello CA, van den Berg WB (2009) Inflammatory arthritis in caspase 1 gene-deficient mice: contribution of proteinase 3 to caspase 1 -independent production of bioactive interleukin-1beta. Arthritis Rheum 60:3651-3662

24. He Z, Bateman A (2003) Progranulin (granulin-epithelin precursor, PC-cell-derived growth factor, acrogranin) mediates tissue repair and tumorigenesis. J Mol Med 81:600-612

25. Zhu J, Nathan C, Jin W, Sim D, Ashcroft GS, Wahl SM, Lacomis L, Erdjument-Bromage H, Tempst P, Wright CD, Ding A (2002) Conversion of proepithelin to epithelins: roles of SLPI and elastase in host defense and wound repair. Cell 111:867-878

26. Kessenbrock K, Fröhlich L, Sixt M, Lämmermann T, Pfister H, Bateman A, Belaaouaj A, Ring J, Ollert M, Fässler R, Jenne DE (2008) Proteinase 3 and neutrophil elastase enhance inflammation in mice by inactivating antiinflammatory progranulin. J Clin Invest 118:2438-2447

27. Cruts M, Van Broeckhoven C (2008) Loss of progranulin function in frontotemporal lobar degeneration. Trends Genet 24:186-194

28. Yin F, Banerjee R, Thomas B, Zhou P, Qian L, Jia T, Ma X, Ma Y, Iadecola C, Beal MF, Nathan C, Ding A (2010) Exaggerated inflammation, impaired host defense, and neuropathology in progranulin-deficient mice. J Exp Med 207:117-128

29. Raptis SZ, Shapiro SD, Simmons PM, Cheng AM, Pham CT (2005) Serine protease cathepsin G regulates adhesion-dependent neutrophil effector functions by modulating integrin clustering. Immunity 22:679-691

30. Uehara A, Muramoto K, Takada H, Sugawara S (2003) Neutrophil serine proteinases activate human nonepithelial cells to produce inflammatory cytokines through protease-activated receptor 2. J Immunol 170:5690-5696

31. Chin AC, Lee WY, Nusrat A, Vergnolle N, Parkos CA (2008) Neutrophil-mediated activation of epithelial protease-activated receptors- 1 and -2 regulates barrier function and transepithelial migration. J Immunol 181:5702-5710

32. Devaney JM, Greene CM, Taggart CC, Carroll TP, O'Neill SJ, McElvaney NG (2003) Neutrophil elastase up-regulates interleukin8 via toll-like receptor 4. FEBS Lett 544:129-132

33. Carp H, Janoff A (1980) Potential mediator of inflammation. Phagocyte-derived oxidants suppress the elastase-inhibitory capacity of alpha 1-proteinase inhibitor in vitro. J Clin Invest 66:987-995 